\title{
Outcomes of Isolated Tricuspid Valve Surgery
}

\author{
Gemma Sánchez-Espín, ${ }^{1}$ Jorge Rodríguez-Capitán, ${ }^{1}$ Juan J. Otero-Forero, ${ }^{1}$ Víctor M. Becerra-Muñoz, ${ }^{1}$ \\ Emiliano A. Rodríguez-Caulo, ${ }^{2}$ Miguel Such-Martínez, ${ }^{1}$ Carlos Porras-Martín, ${ }^{1}$ José M. Villaescusa-Catalán, ${ }^{1}$ \\ María J. Mataró-López, ${ }^{1}$ Arantza Guzón-Rementería, ${ }^{1}$ José M. Melero-Tejedor, ${ }^{1}$ \\ Encarnación Gutierrez-Carretero, ${ }^{3}$ Manuel F. Jiménez-Navarro ${ }^{1}$
}

${ }^{1}$ Unidad de Gestión Clínica del Corazón, Hospital Virgen de la Victoria de Málaga, Málaga, Spain, Instituto Biosanitario Málaga (IBIMA), CIBERCV Málaga. Universidad de Málaga (UMA), Málaga, Spain; ${ }^{2}$ Servicio de Cirugía Cardiovascular, Hospital Virgen Macarena, Sevilla, Spain; ${ }^{3}$ Servicio de Cirugía Cardiovascular, Hospital Virgen del Rocío, Sevilla, Spain, CIBERCV Sevilla, Spain

\section{ABSTRACT}

Background: Isolated tricuspid valve surgery is a rarely performed procedure and traditionally is associated with a bad prognosis, although its clinical outcomes still are little known. The aim of this study was to assess the short- and long-term clinical outcomes obtained at our center after isolated tricuspid valve surgery as treatment for severe tricuspid regurgitation.

Methods: This retrospective study included 71 consecutive patients with severe tricuspid regurgitation who underwent isolated tricuspid valve surgery between December 1996 and December 2017. Perioperative and long-term mortality, tricuspid valve reoperation, and functional class were analyzed after follow up.

Results: Regarding surgery, $7 \%$ of patients received a De Vega annuloplasty, $14.1 \%$ an annuloplasty ring, $11.3 \%$ a mechanical prosthesis, and $67.6 \%$ a biological prosthesis. Perioperative mortality was $12.7 \%$ and no variable was shown to be predictive of this event. After a median follow up of 45.5 months, long-term mortality was $36.6 \%$, and the multivariate analysis identified atrial fibrillation as the only predictor (Hazard Ratio 3.014, 95\% confidence interval 1.06-8.566; $P=0.038)$. At the end of follow up, $63.6 \%$ of survivors had functional class I.

Conclusions: Isolated tricuspid valve surgery was infrequent in our center. Perioperative mortality was high, as was long-term mortality. However, a high percentage of survivors were barely symptomatic after follow up.

\section{INTRODUCTION}

The tricuspid valve has been dubbed the "forgotten valve" as, both clinically and in terms of research, tricuspid

Received May 26, 2020; accepted fune 25, 2020.

Correspondence: forge Rodríguez-Capitán, Cardiology, Hospital University Virgen de la Victoria, Campus de Teatinos S/N 29010, $5^{a}$ Planta, Málaga, Spain; +34-951032054; fax: +34-951032560 (e-mail: capijorge@hotmail.com); Gemma Sánchez-Espín, Cardiovascular Surgery, Hospital University Virgen de la Victoria, Campus de Teatinos S/N 29010, $5^{a}$ Planta, Málaga, Spain; +34951032054; fax: +34-951032560 (e-mail: gemmase@hotmail.com). pathology has received less attention than left valve disease [Shah 2008]. Nevertheless, it is currently recognized that tricuspid regurgitation is not a benign entity, as patients with severe tricuspid regurgitation, whether isolated or associated with other pathologies, present higher morbidity and mortality than the general population [Nath 2004; Topilsky 2019]. Despite this change of paradigm, isolated tricuspid valve surgery is not frequently performed, in great measure due to the bad short- and long-term outcomes observed in the initial experiences after the surgical procedure, particularly among patients with a history of previous heart surgery [Baumgartner 2017]. These bad outcomes represent a vicious circle for the management of severe tricuspid regurgitation because the patients are initially treated with a conservative strategy and are only referred for surgical treatment when they reach a clinically advanced stage, when surgical outcomes can mean it is inadvisable [Topilsky 2011]. In recent years, as a response to this circumstance, different percutaneous therapies have emerged to treat tricuspid regurgitation which offer good results, at least in the shortand mid-term [Taramasso 2019]. In this context, it is of particular interest to broaden our knowledge of the clinical outcomes of isolated tricuspid valve surgery, as the information available to date is insufficient and fundamentally based on the few studies, which have small samples, or on the sparse registries in existence, which also have constraints [Fender 2018]. For these reasons, the aim of this study was to assess the short- and long-term clinical outcomes obtained at our center after isolated tricuspid valve surgery as the treatment for severe tricuspid regurgitation.

\section{MATERIALS AND METHODS}

Patients: We designed a retrospective single-center, observational study that included all of the consecutive patients with severe tricuspid regurgitation who underwent isolated tricuspid valve replacement or repair surgery in our center between December 1996 and December 2017. Patients who also underwent another concomitant surgical procedure at the time of tricuspid valve surgery were excluded. The indication for tricuspid surgery was established by presentation of severe symptomatic tricuspid regurgitation despite medical 


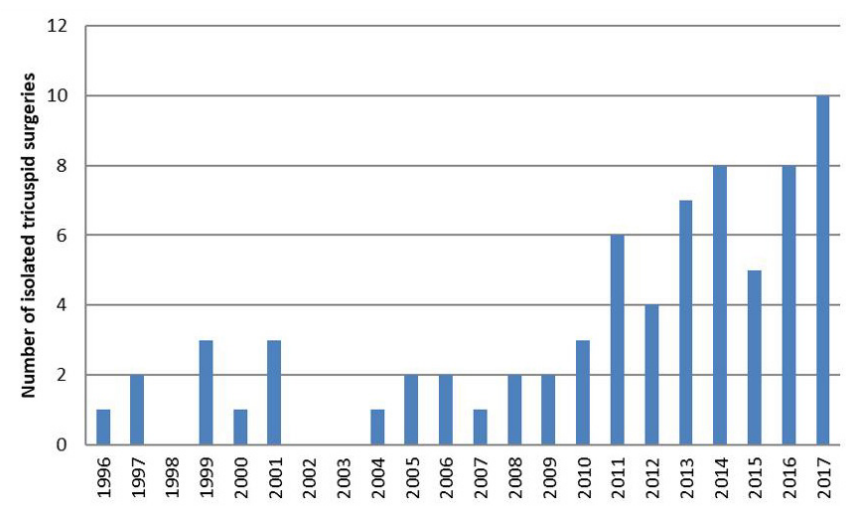

Figure 1. Number of isolated tricuspid valve surgeries performed each year.

treatment. During the stated inclusion period, a total of 330 patients underwent tricuspid valve surgery. Of these, 71 $(21.5 \%)$ underwent isolated tricuspid valve surgery and were included in this study.

Data collection: All of the patient clinical and surgical data were compiled from their medical records. The baseline (before surgery) variables collected were age, gender, diabetes mellitus, arterial hypertension, chronic obstructive pulmonary disease, kidney failure (defined as creatinine clearance lower than $30 \mathrm{ml} / \mathrm{min}$ ), liver disease (defined as the presence of echographic signs of liver involvement or higher than normal transaminase values), atrial fibrillation, previous pacemaker implant, organic or functional etiology of the tricuspid regurgitation, and presence or not of previous heart surgery.

With regard to echocardiographic data, tricuspid regurgitation was defined as severe in the presence of a regurgitant flow with an area greater than $30 \%$ of the area of the right atrium, insufficient valve coaptation and the presence of reverse systolic flow in the hepatic vein [Zoghbi 2003]. Pulmonary artery systolic pressure was estimated from tricuspid regurgitation peak velocity and classified as pulmonary hypertension, where pulmonary artery systolic pressure was higher than $50 \mathrm{mmHg}$. Systolic function of the left ventricle was assessed in accordance with American Society of Echocardiography recommendations, and cases with a left ventricular ejection fraction lower than $55 \%$ were classified as left ventricle systolic dysfunction [Lang 2005].

Information was collected about type of surgical repair or tricuspid valve replacement, extracorporeal circulation time, and the performance of surgery with aortic clamping or beating heart. The type of surgical procedure (De Vega annuloplasty, ring annuloplasty, implantation of mechanical prosthesis or of biological prosthesis) was decided in each case by consensus between the surgeon, cardiologist, and patient. All the interventions were conducted with extracorporeal circulation and full median sternotomy or right mini-thoracotomy. Both conventional central cannulation of the ascending aorta and venae cavae and peripheral cannulation via the femoral veins with full herapinization were performed. The decision to carry out the procedure using aortic clamp and cardioplegia, or without clamping (beating heart),

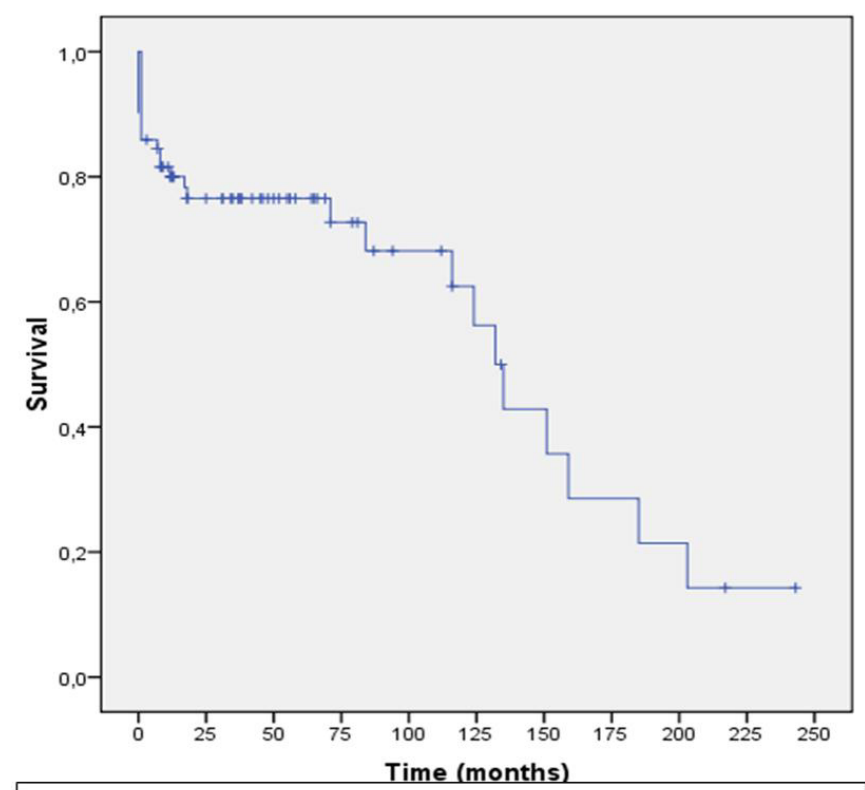

Number at risk:

$\begin{array}{llllllllll}71 & 41 & 28 & 18 & 13 & 9 & 6 & 4 & 3 & 1\end{array}$

Figure 2. Kaplan-Meier survival curve after long-term follow up.

was made according to patient characteristics and surgeon preference. In May 2013, 50\% of the total number of isolated tricuspid valve surgical procedures in this series was reached. This date was therefore set as the cut-off point for analyzing the clinical outcomes both for the preceding and the subsequent periods.

During follow up, we assessed perioperative mortality (death for any cause in the 30 days subsequent to tricuspid surgery or during hospital admission for said surgery) longterm mortality, repeat tricuspid surgery, and the New York Heart Association functional class of survivors at the end of follow up.

Statistical analysis: Statistical analysis was conducted using SPSS 19.0 statistics software. Qualitative variables are expressed both in absolute numbers and as a percentage and were compared using the Chi-squared test or Fischer's exact test. Follow-up time is described as a median and interquartile range, and the other quantitative variables as a mean \pm standard deviation, and according to their distribution were compared with Student's t-test or the MannWhitney-Wilcoxon $U$ test. Distribution of the variables was evaluated by means of the Kolmogorov-Smirnov test. A univariate analysis of perioperative mortality was performed. In order to evaluate predictors of mortality after follow up, a univariate analysis initially was conducted. After that, parameters with a $P$ value $\leq .05$ from the univariate analysis were included in a Cox model to identify the independent variables predictive of total mortality and to calculate the Hazard Ratio and corresponding $95 \%$ confidence interval. Two-tailed values of $P \leq .05$ were considered significant for each of these tests. In addition, survival analysis followed the Kaplan-Meier method. 
Table 1. Clinical and echocardiographic baseline characteristics, and surgical data

\begin{tabular}{|c|c|c|c|c|}
\hline Age (years) & $61.5 \pm 12.3$ & $65.1 \pm 9.4$ & $54.1 \pm 14.4$ & .002 \\
\hline Arterial hypertension & $33(46.5 \%)$ & $26(54.2 \%)$ & $7(30.4 \%)$ & .061 \\
\hline Diabetes mellitus & $12(16.9 \%)$ & $7(14.6 \%)$ & $5(21.7 \%)$ & .507 \\
\hline Previous pacemaker implant & $4(5.6 \%)$ & $3(6.3 \%)$ & $1(4.3 \%)$ & 1 \\
\hline COPD & $12(16.9 \%)$ & $10(20.8 \%)$ & $2(8.7 \%)$ & .313 \\
\hline Liver disease & $6(8.5 \%)$ & $3(6.3 \%)$ & $3(13 \%)$ & .381 \\
\hline Kidney failure & $9(12.7 \%)$ & $6(12.5 \%)$ & $3(13 \%)$ & 1 \\
\hline Previous heart surgery & $46(64.8 \%)$ & $34(70.8 \%)$ & $12(52.2 \%)$ & .123 \\
\hline \multicolumn{5}{|l|}{ Surgical data } \\
\hline Tricuspid repair & $15(21.1 \%)$ & $11(22.9 \%)$ & $4(17.4 \%)$ & .759 \\
\hline Tricuspid replacement & $56(78.9 \%)$ & $37(77.1 \%)$ & $19(82.6 \%)$ & .759 \\
\hline De Vega annuloplasty & $5(7 \%)$ & $3(6.3 \%)$ & $2(8.7 \%)$ & .656 \\
\hline Ring annuloplasty & $10(14.1 \%)$ & $8(16.7 \%)$ & $2(8.7 \%)$ & .482 \\
\hline Mechanical prosthesis & $8(11.3 \%)$ & $2(4.2 \%)$ & $6(26.1 \%)$ & .012 \\
\hline Biological prosthesis & $48(67.6 \%)$ & $35(72.9 \%)$ & $13(56.5 \%)$ & .134 \\
\hline ECC time (min) & $85.7 \pm 51.5$ & $84.4 \pm 58.1$ & $88.2 \pm 35$ & .731 \\
\hline Beating heart surgery & $56(78.9 \%)$ & $40(83.3 \%)$ & $16(69.6 \%)$ & .221 \\
\hline
\end{tabular}

Data are expressed as $\mathrm{n}(\%)$ or mean \pm standard deviation

COPD: chronic obstructive pulmonary disease; ECC: extracorporeal circulation; LVEF: left ventricular ejection fraction; PASP: pulmonary artery systolic pressure

\section{RESULTS}

Baseline characteristics: Table 1 shows the clinical and echocardiographic baseline characteristics of the sample, and data about the surgical procedure (Table 1). The mean age of the sample was $61.5 \pm 12.3$ years and $62 \%$ were women. After comparing functional etiology and organic etiology, patients with functional regurgitation were older, had atrial fibrillation more frequently, and received mechanical tricuspid prosthesis less commonly. Among the cases of organic tricuspid disease, six were of rheumatic origin, five secondary to infective endocarditis, three congenital (Ebstein's anomaly), two due to prolapse, two were secondary to carcinoid syndrome, two secondary to pacemaker leads, and three were of undetermined origin. Of the cases, $64.8 \%$ had undergone previous heart surgery; Table 2 summarizes the characteristics of the previous surgery (Table 2).

Regarding isolated tricuspid valve surgery, $7 \%$ of patients underwent a De Vega procedure, $14.1 \%$ had an annuloplasty ring, $11.3 \%$ a mechanical prosthesis, and $67.6 \%$ a biological prosthesis; $78.9 \%$ of patients underwent beating heart surgery. Figure 1 presents the distribution of the number of isolated tricuspid valve surgeries performed each year, which increased predominantly since 2010, reaching the maximum of 10 procedures in 2017 (Figure 1).

Surgical outcomes: Perioperative mortality was recorded at $12.7 \%$ (nine patients). Table 3 shows the univariate analysis of perioperative mortality, in which none of the analyzed variables reached statistical significance, so the subsequent multivariate analysis was not conducted (Table 3). Perioperative mortality in the period before May 2013 was $17.1 \%$, whereas this figure was $8.3 \%$ for procedures performed after that date, although these differences did not reach statistical significance $(P=.307)$.

Long-term clinical follow up: Clinical follow up in terms of mortality and of the need or not for a repeat procedure on the tricuspid valve was completed for $100 \%$ of patients who received hospital discharge (median follow up of 45.5 months, interquartile range 13-84.75 months). In addition, at the end of the long-term follow up, the New York Heart Association functional class of $97.8 \%$ of the follow-up survivors was recorded.

After the perioperative period, 17 patients died, so total mortality after long-term follow up was 36.6\% (26 patients). Figure 2 shows the Kaplan-Meier survival curve after 
Table 2. Cardiac surgeries performed prior to the isolated tricuspid valve surgery (total 46 operations)

\begin{tabular}{lc}
\hline Type of surgery & Number \\
\hline Mechanical mitral prosthesis alone & 23 \\
Mitral repair alone & 1 \\
Mechanical mitral prosthesis and mechanical aortic prosthesis & 8 \\
Mechanical aortic prosthesis alone & 1 \\
Biological aortic prosthesis and mitral repair & 1 \\
Mechanical mitral prosthesis, mechanical aortic prosthesis and & 1 \\
mechanical tricuspid prosthesis & 2 \\
Mechanical mitral prosthesis and tricuspid repair & 1 \\
Tricuspid valvulectomy without replacement alone & 2 \\
Mechanical tricuspid prosthesis alone & 1 \\
Tricuspid repair alone & 4 \\
Non-tricuspid related congenital disease surgery & 1 \\
Endocardial lead extraction & 1
\end{tabular}

long-term follow up (Figure 2). Table 4 shows the univariate analysis of total mortality after follow up, and the only variables that showed a statistically significant association with that event were atrial fibrillation (Hazard Ratio 3.251, 95\% confidence interval 1.163-9.086, $P=.025)$ and a left ventricular ejection fraction less than 55\% (Hazard Ratio 4.309, 95\% confidence interval 1.226-15.14, $P=.023$ ) (Table 4). These two variables were included in a multivariate analysis that identified the presence of atrial fibrillation (Hazard Ratio $3.014,95 \%$ confidence interval $1.06-8.566, P=.038)$ as a predictor of total mortality after follow up but not a left ventricular ejection fraction less than 55\% (Hazard Ratio 3.347, 95\% confidence interval 0.944-11.861, $P=.061)$.

During follow up, four patients $(6.4 \%$ of the survivors of the perioperative period) underwent a further surgical intervention on the tricuspid valve. Two patients suffered mechanical prosthetic thrombosis and in both cases had a biological prosthesis implanted. The other two reoperations also were biological prosthesis implantations, which were indicated after the observation of severe symptomatic tricuspid regurgitation in patients who previously had undergone tricuspid repair, one with the De Vega technique and the other with a tricuspid annulus. Regarding New York Heart Association functional class at the end of follow up, $63.6 \%$ of patients were in functional class I, $18.2 \%$ in functional class II, and $18.2 \%$ in functional class III.

\section{DISCUSSION}

This work shows the short- and long-term clinical outcomes of a series of patients with severe tricuspid regurgitation who underwent isolated surgery on the tricuspid valve. Perioperative and long-term mortality were high in comparison with the mortality figures classically reported for left valve surgery [Gammie 2009; Kaneki 2015].
Table 3. Univariate analysis of perioperative mortality

\begin{tabular}{|c|c|c|c|}
\hline & $\begin{array}{l}\text { Survivors } \\
(\mathrm{N}=62)\end{array}$ & $\begin{array}{c}\text { Deceased } \\
(N=9)\end{array}$ & $P$ \\
\hline Age (years) & $61.2 \pm 12.5$ & $63.7 \pm 10$ & .337 \\
\hline Female gender & $39(62.9 \%)$ & $5(55.6 \%)$ & .723 \\
\hline Arterial hypertension & $30(48.4 \%)$ & $3(33.3 \%)$ & .489 \\
\hline Diabetes mellitus & $10(16.1 \%)$ & $2(22.2 \%)$ & .643 \\
\hline Atrial fibrillation & $43(69.4 \%)$ & $7(77.8 \%)$ & .716 \\
\hline Previous pacemaker implant & $3(4.8 \%)$ & $1(11.1 \%)$ & .426 \\
\hline COPD & $9(14.5 \%)$ & $3(33.3 \%)$ & .172 \\
\hline Liver disease & $6(9.7 \%)$ & $0(0 \%)$ & 1 \\
\hline Kidney failure & $8(12.9 \%)$ & $1(11.1 \%)$ & 1 \\
\hline Functional etiology & $41(68.3 \%)$ & $5(62.5 \%)$ & .707 \\
\hline Previous heart surgery & $40(64.5 \%)$ & $6(66.7 \%)$ & 1 \\
\hline LVEF $<55 \%$ & $3(4.8 \%)$ & $1(11.1 \%)$ & .426 \\
\hline PASP $>50 \mathrm{mmHg}$ & $25(40.3 \%)$ & $5(55.6 \%)$ & .479 \\
\hline Tricuspid repair & $13(21 \%)$ & $2(22 \%)$ & 1 \\
\hline Tricuspid replacement & $49(79 \%)$ & $7(77.8 \%)$ & 1 \\
\hline De Vega annuloplasty & $5(8.1 \%)$ & $0(0 \%)$ & .52 \\
\hline Ring annuloplasty & $8(12.9 \%)$ & $2(22.2 \%)$ & .52 \\
\hline Mechanical prosthesis & $7(11.3 \%)$ & $1(11.1 \%)$ & 1 \\
\hline Biological prosthesis & $42(67.7 \%)$ & $6(66.6 \%)$ & 1 \\
\hline ECC time (min) & $81.8 \pm 43.5$ & $112.3 \pm 88.5$ & .523 \\
\hline Beating heart surgery & $47(75.8 \%)$ & $9(100 \%)$ & .189 \\
\hline Surgery from May 2013 onward & $33(53.2 \%)$ & $3(33.3 \%)$ & .307 \\
\hline
\end{tabular}

Data are expressed as $\mathrm{n}(\%)$ or mean \pm standard deviation COPD: chronic obstructive pulmonary disease; ECC: extracorporeal circulation; LVEF: left ventricular ejection fraction; PASP: pulmonary artery systolic pressure

In our series, after a lengthy inclusion period of 21 years, only 71 patients with severe tricuspid regurgitation underwent isolated tricuspid surgery, which is only $21.5 \%$ of the total number of patients undergoing tricuspid valve procedures. The situation was, however, very different in the final years of our study, when between five and 10 procedures were performed per year, compared with the first years, when zero to three surgeries were performed annually. The data published by other centers and in the extensive national registries show that isolated tricuspid valve surgery has, in general, been uncommon to date as tricuspid surgery predominantly has been associated with left valve intervention and, in parallel with what is shown in our research, in recent years there has been a marked increase in the number of isolated tricuspid valve surgical procedures [Zack 2017; Alqahtani 2017; Kundi 2019]. With regard to surgical procedure, the predominant choice in our series was beating heart surgery (78.9\% of cases) and for tricuspid valve replacement with biological prosthesis $(67.6 \%$ of cases). This again shows a parallel with other 
Table 4. Univariate analysis of total mortality after follow up

\begin{tabular}{lcc}
\hline & Total mortality & $P$ \\
\hline Age (years) & $1.03(0.996-1.066)$ & .088 \\
Female gender & $0.779(0.347-1.749)$ & .545 \\
Arterial hypertension & $1.187(0.53-2.657)$ & .677 \\
Diabetes mellitus & $2.119(0.82-5.474)$ & .121 \\
Atrial fibrillation & $3.251(1.163-9.086)$ & .025 \\
Previous pacemaker implant & $1.148(0.152-8.699)$ & .894 \\
COPD & $1.949(0.71-5.348)$ & .195 \\
Liver disease & $1.637(0.373-7.186)$ & .513 \\
Kidney failure & $1.886(0.693-5.128)$ & .214 \\
Functional etiology & $1.306(0.534-3.197)$ & .558 \\
Previous heart surgery & $1.813(0.693-4.742)$ & .225 \\
LVEF < 55\% & $4.309(1.226-15.14)$ & .023 \\
PASP > 50 mmHg & $1.641(0.75-3.589)$ & .215 \\
Tricuspid repair & $1.436(0.63-3.277)$ & .39 \\
Tricuspid replacement & $0.696(0.305-1.588)$ & .39 \\
ECC time (min) & $1.006(0.999-1.013)$ & .1 \\
Beating heart surgery & $2.061(0.779-5.45)$ & .145 \\
Surgery from May 2013 onwards & $1.022(0.382-2.733)$ & .965 \\
\hline
\end{tabular}

Data are expressed as Hazard Ratio ( $95 \%$ confidence interval). COPD: chronic obstructive pulmonary disease; ECC: extracorporeal circulation; LVEF: left ventricular ejection fraction; PASP: pulmonary artery systolic pressure

series and the aforementioned large registries, which indicate that, although repair predominates over valve replacement in tricuspid surgery globally, in isolated tricuspid surgery, valve replacement is the most frequent procedure [Zack 2017; Alqahtani 2017; Kundi 2019; Ejiofor 2017]. Regarding the clinical profile of our patients, we observed two groups: On the one hand, patients with functional tricuspid regurgitation, who were older and also were more likely to have atrial fibrillation, and on the other hand, patients with organic etiology, who received mechanical tricuspid prosthesis more frequently, probably in relation to their younger age. The functional tricuspid group was larger, as it also happens with other series on isolated tricuspid surgery [De Meester 2014; Lee 2010].

Perioperative mortality was $12.7 \%$, a figure that should be considered high in comparison with the outcomes of left valve surgery [Gammie 2009; Kaneko 2015]. The majority of the few series published with data on isolated tricuspid surgery have shown suboptimal perioperative outcomes, on occasion with mortality figures of $20 \%$ [Mangoni 2001] or $16 \%$ [Guenther 2008], or in other instances with somewhat better figures than those shown in our research, such as $7.9 \%$ [De Meester 2014] or 8.8\% [Lee 2010]. Chen et al published interesting outcomes, with no cases of perioperative mortality in a series of 28 patients undergoing isolated tricuspid surgery with a minimally invasive, beating-heart endoscopic procedure [Chen 2019]. The outcomes of three extensive contemporary national registries from the United States, which report an 8.8\% [Zack 2017], 8.1\% [Alqahtani 2017] and $9.9 \%$ [Kundi 2019] of perioperative mortality, are also particularly interesting. Contemporary series recently published can be illustrative examples of current outcomes after isolated tricuspid valve surgery, and they have shown better perioperative results than the results reported in this manuscript. We emphasize studies showing perioperative mortality as $8.5 \%$ [Chen 2020], 3.4\% [Liu 2019], or 3.1\% [Lu 2020]. In order to achieve positive outcomes, all these studies coincide with the implementation of minimally invasive surgical techniques. Furthermore, we hypothesize that an early selection of patients could help to achieve the low perioperative mortality observed because patients in the aforementioned series had mean ages of 55.8 [Chen 2020], 56.4 [Liu 2019], and 57.4 years [ $\mathrm{Lu} 2020]$, all of them under the mean age of the sample of our study.

In recent years, various percutaneous techniques have been developed that have shown their feasibility by offering acceptable perioperative results after tricuspid regurgitation treatment [Taramasso 2019]. These emerging percutaneous therapies are promising, but are still in their initial phases of development and require further studies that provide more information on the indications and the optimum techniques, as well as the long-term outcomes. Our research did not find any predictor of perioperative mortality, which can be explained, at least in part, by its low statistical power, due to the small size of our sample. So despite the fact that both classically and in recent publications replacement with prosthesis has been associated with greater perioperative mortality and worse outcomes during follow up, in our study, replacement with prosthesis was not associated with higher perioperative mortality [Zack 2017; Guenther 2008]. Likewise, the decrease in perioperative mortality observed from May 2013 (17.1\% compared with 8.3\%) did not reach statistical significance. Our group has previously published its global results for tricuspid surgery (both isolated and associated with another procedure) in two papers: The first evaluated the patients operated on between April 1996 and February 2010, while the second included the patients undergoing procedures between March 2010 and December 2013 [Rodríguez-Capitán 2013; Becerra-Muñoz 2019]. Perioperative mortality went from $18.5 \%$ in the older series to $8 \%$ in the more recent, in parallel with this paper's findings on isolated tricuspid surgery. The causes that explain the trend toward better perioperative mortality in our series are unknown. It is possible that different anesthetic and surgical techniques used over time could have influenced perioperative outcomes. In addition to this, the indication for surgical treatment could be established in earlier clinical stages, in accordance with studies that have shown worse outcomes in patients operated on after developing advanced symptoms [Topilsky 2011]. With regard to the trend that perioperative mortality after isolated tricuspid valve surgery has followed over time, large registries do not show a significant decrease over time in recent years, despite a significant increase in the 
number of these surgical procedures that is performed annually [Zack 2017; Alqahtani 2017].

During the follow up of our series, we observed a mortality that we consider high (36.6\% of patients); on the other hand, the majority of survivors at the end of the follow up barely were symptomatic, 63.6\% with New York Heart Association class I and $18.2 \%$ with class II. Although the information available about the long-term outcomes of isolated tricuspid valve surgery is still limited, the two circumstances observed in our study, a not insignificant mortality during follow up alongside good functional class outcomes for survivors, has previously been described in various series on isolated tricuspid valve surgery [Pfannmüller 2012; Kim 2009; Kim 2013]. In our research, the only predictor associated with mortality in the long term was a preoperative diagnosis of atrial fibrillation. The mechanism by which this association could exist is uncertain, but we can put forward some hypotheses: First, atrial fibrillation conditions an increase in morbidity and mortality under any circumstances; on the other hand, in the context of tricuspid regurgitation, the development of atrial fibrillation could be precipitated by greater pressure in the right atrium. This increase in right atrium pressure has been shown to be a predictor of perioperative mortality after tricuspid surgery [Kuwaki 2001] and also has been related with long-term mortality after the surgical treatment of tricuspid regurgitation [Topilsky 2011].

In the series we present here, the percentage of reoperations $(6.4 \%)$ on the tricuspid valve may be considered comparable to that observed in other studies on isolated tricuspid valve surgery [Pfannmüller 2012]. It should be noted that, in our series, of the seven patients who received a mechanical tricuspid implantation and survived the perioperative period, two $(28.5 \%)$ underwent a tricuspid reoperation, compared with the group that received a biological prosthesis, who had no tricuspid reoperations. Due to the bad outcomes associated with mechanical prosthesis, in our center no mechanical prosthesis has been implanted in isolated tricuspid surgery since the year 2006. Nevertheless, these data contrast with those of a broad meta-analysis of 2,630 patients who underwent implantation of a tricuspid prosthesis, which did not find significant differences between mechanical and biological prosthesis in terms of reoperation during follow up. That meta-analysis did find a higher risk of prosthesis thrombosis in the group treated with mechanical prosthesis [Liu 2016].

This work presents the limitations associated with a retrospective, single center study. Despite a prolonged inclusion period, the sample size was small, which substantially limits the likelihood of identifying predictors of clinical events. The etiology of tricuspid valvular heart disease was heterogeneous, which makes generalization of the observed results difficult. The clinical results also could have been influenced by the variability of surgical technique and the management of perioperative anaesthetic. Finally, another limitation is the absence of data, due to their not being available in the medical records, probably related to clinical events, such as those related to New York Heart Association functional class before surgery, and the parameters of right ventricle volume and function with imaging techniques.
In conclusion, in our center, isolated tricuspid valve surgery has globally been infrequent, although in recent years it has become more frequent. Perioperative mortality was high, as was long-term mortality. Nevertheless, the majority of the survivors at the end of follow up were barely symptomatic and had New York Heart Association functional class I or II.

\section{REFERENCES}

Alqahtani F, Berzingi CO, Aljohani S, Hijazi M, Al-Hallak A, Alkhouli M. 2017. Contemporary Trends in the Use and Outcomes of Surgical Treatment of Tricuspid Regurgitation. J Am Heart Assoc 6. pii: e007597.

Baumgartner H, Falk V, Bax JJ, et al. 2017. ESC Scientific Document Group. 2017 ESC/EACTS Guidelines for the management of valvular heart disease. Eur Heart J 38:2739-91.

Becerra-Muñoz VM, Rodríguez-Capitán J, Sánchez-Espín G, SuchMartínez M, Gómez-Doblas JJ, de Teresa-Galván E. 2019. Outcomes After Surgical Treatment of Severe Tricuspid Regurgitation in a Contemporary Series. Rev Esp Cardiol (Engl Ed) 72:178-80.

Chen J, Hu K, Ma W, et al. 2020. Isolated reoperation for tricuspid regurgitation after left-sided valve surgery: technique evolution. Eur J Cardiothorac Surg 5:142-150.

Chen Z, Ke Y, Xie X, et al. 2019. Beating-Heart Totally Endoscopic Tricuspid Valvuloplasty in Reoperative Cardiac Surgery. Ann Thorac Surg 107:e79-e82.

De Meester P, Van De Bruaene A, Voigt J-U, Herijgers P, Budts W. 2014. Outcome and determinants of prognosis in patients undergoing isolated tricuspid valve surgery: Retrospective single center analysis. Int J Cardiol 175:333-9.

Ejiofor JI, Neely RC, Yammine M, et al. 2017. Surgical outcomes of isolated tricuspid valve procedures: repair versus replacement. Ann Cardiothorac Surg 6:214-22.

Fender EA, Zack CJ, Nishimura RA. 2018. Isolated tricuspid regurgitation: outcomes and therapeutic interventions. Heart 104:798-806.

Gammie JS, Sheng S, Griffith BP, et al. 2009. Trends in mitral valve surgery in the United States: results from the Society of Thoracic Surgeons Adult Cardiac Surgery Database. Ann Thorac Surg 87:1431-37.

Guenther T, Noebauer C, Mazzitelli D, Busch R, Tassani-Prell P, Lange R. 2008. Tricuspid valve surgery: a thirty-year assessment of early and late outcome. Eur J Cardiothorac Surg 34:402-9.

Kaneko T, Vassileva CM, Englum B, et al. 2015. Contemporary outcomes of repeat aortic valve replacement: a benchmark for transcatheter valve-in-valve procedures. Ann Thorac Surg 100:1298-1304.

Kim JB, Jung SH, Choo SJ, Chung CH, Lee JW. 2013. Clinical and echocardiographic outcomes after surgery for severe isolated tricuspid regurgitation. J Thorac Cardiovasc Surg 146:278-84.

Kim YJ, Kwon DA, Kim HK, et al. 2009. Determinants of surgical outcome in patients with isolated tricuspid regurgitation. Circulation 120:1672-78.

Kundi H, Popma JJ, Cohen DJ, et al. 2019. Prevalence and Outcomes of Isolated Tricuspid Valve Surgery Among Medicare. Am J Cardiol 123:132-8.

Kuwaki K, Morishita K, Tsukamoto M, Abe T. 2001. Tricuspid valve surgery for functional tricuspid valve regurgitation associated with left-sided valvular disease. Eur J Cardiothorac Surg 20:577-82. 
Lang RM, Bierig M, Devereux BB, et al. Chamber Quantification Writing Group; American Society of Echocardiography's Guidelines and Standards Committee; European Association of Echocardiography. 2005. Recommendations for chamber quantification: a report from the American Society of Echocardiography's Guidelines and Standards Committee and the Chamber Quantification Writing Group, developed in conjunction with the European Association of Echocardiography, a branch of $\geq$ the European Society of Cardiology. J Am Soc Echocardiogr 18:1440-63.

Lee JW, Song JM, Park JP, Lee JW, Kang DH, Song JK. 2010. LongTerm Prognosis of Isolated Significant Tricuspid Regurgitation. Circ $\mathrm{J} ; 74: 375-80$.

Liu P, Qiao WH, Sun FQ, et al. 2016. Should a Mechanical or Biological Prosthesis Be Used for a Tricuspid Valve Replacement? A Meta-Analysis. J Card Surg 31:294-302.

Liu S, Chen JM, Wang WS, et al. 2019. Short-term outcomes of minimally invasive reoperation for tricuspid regurgitation after left-sided valve surgery. Zhonghua Wai Ke Za Zhi. 57:898-901.

Lu S, Song K, Yao W, et al. 2020. Simplified, minimally invasive, beatingheart technique for redo isolated tricuspid valve surgery. Version 2.J Cardiothorac Surg;15:146.

Mangoni AA, DiSalvo TG, Vlahakes GJ, Polanczyk CA, Fifer MA. 2001. Outcome following isolated tricuspid valve replacement. Eur J Cardiothorac Surg 19:68-73.

Nath J, Foster E, Heidenreich PA. 2004. Impact of tricuspid regurgitation on long-term survival. J Am Coll Cardiol 43:405-9.
Pfannmüller B, Misfeld M, Borger MA, et al. 2012. Isolated reoperative minimally invasive tricuspid valve operations. Ann Thorac Surg 94:2005-10.

Rodríguez-Capitán J, Gómez-Doblas JJ, Fernández-López L, et al. Short- and long-term outcomes of surgery for severe tricuspid regurgitation. 2013. Rev Esp Cardiol (Engl Ed) 66:629-35.

Shah PM, Raney AA. 2008. Tricuspid Valve Disease. Curr Probl in Cardiol 3:47-84.

Taramasso M, Alessandrini H, Latib A, et al. 2019. Outcomes After Current Transcatheter Tricuspid Valve Intervention: Mid-Term Results From the International TriValve Registry. JACC Cardiovasc Interv 12:155-65.

Topilsky Y, Khanna AD, Oh JK, et al. 2011. Preoperative factors associated with adverse outcome after tricuspid valve replacement. Circulation 123:1929-39.

Topilsky Y, Maltais S, Medina Inojosa J, et al. 2019. Burden of Tricuspid Regurgitation in Patients Diagnosed in the Community Setting. JACC Cardiovasc Imaging 12:433-42.

Zack CJ, Fender EA, Chandrashekar P, et al. 2017. National Trends and Outcomes in Isolated Tricuspid Valve Surgery. J Am Coll Cardiol 70:2953-60

Zoghbi WA, Enriquez-Sarano M, Foster E, et al. 2003. American Society of Echocardiography. Recommendations for evaluation of the severity of native valvular regurgitation with two-dimensional and Doppler echocardiography. J Am Soc Echocardiogr 16:777-802. 